We also looked at use of higher dose corticosteroids or IS in the first two years after diagnosis. We assessed whether each of these patterns of drug use varied between different patient groups.

Results Figure 1a shows that $134(78 \%)$ of patients received steroids, 121 (71\%) hydroxychloroquine and 98 (57\%) IS. About a third (59 or 35\%) received all three. Conversely, 33 (19\%) received no drugs or hydroxychloroquine alone. 94\% of those who received IS also got steroids.

There were no significant associations with gender or ethnicity except that a higher proportion of non-white patients than white received both higher dose corticosteroids and IS $(36 \%$ vs $21 \% \mathrm{p}=0.05)$. Figure $1 \mathrm{~b}$ shows that use of most drugs was similar across all ages of onset, except that those diagnosed at $>50$ years had lower use of higher dose corticosteroids (40\%) and azathioprine (10\%) but higher use of cyclophosphamide $(30 \%)$ than other groups.

Figure 1c shows a shift to increased early use of IS and higher dose corticosteroids in patients diagnosed in more recent decades. Cyclophosphamide use has continued despite introduction of rituximab and mycophenolate.

Conclusion This long-term retrospective analysis shows that over the course of disease $81 \%$ of patients received corticosteroids and/or immunosuppressants. Early aggressive immunosuppression has become more common.

\section{PS6:112 ACCEPTANCE AND COMMITMENT THERAPY IN LUPUS, A CASE CONTROL STUDY}

${ }^{1} \mathrm{M}$ Sahebari, ${ }^{2} \mathrm{~A}$ Ahmadi, ${ }^{2} \mathrm{HR}$ Aghamohammadian, ${ }^{2} \mathrm{MJ}$ Asghari, ${ }^{1} \mathrm{SZ}$ Mirfeizi. ${ }^{1}$ Rheumatic Diseases Research Centre, Mashhad University of Medical Sciences, School of Medicine, Mashhad, Iran; ${ }^{2}$ Ferdowsi University of Mashhad, Faculty of Education and Psychology, Mashhad, Iran

10.1136/lupus-2018-abstract.156

The aim of this study was to evaluate the influence of acceptance and commitment therapy (ACT) in lupus, on frustration,
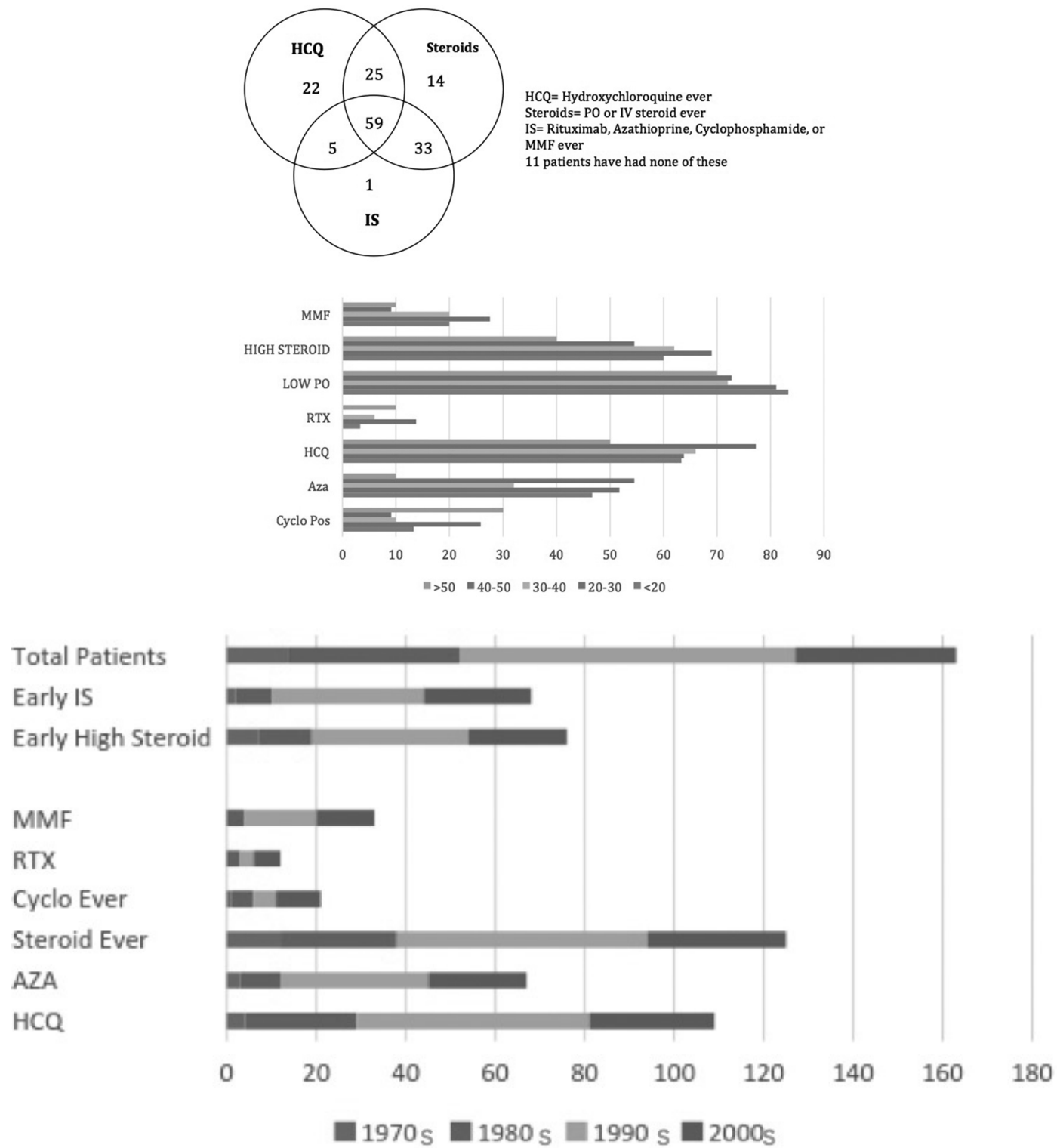

Abstract PS6:111 Figure 1 A: all drugs. B: drugs stratified by age of SLE diagnosis. C: drugs stratified by decade of diagnosis 
psychological distress and mental tiredness. In this study, we compared the results of the pretest-post test evaluation of participants in two groups of lupus patients with and without intervention. 24 lupus patients participated in this study. Then they divided randomly into two groups including patients with ACT intervention and patients without intervention. Both groups were matched for age, major organ involvement, education and economic level. At the beginning and end of the study, all patients filled out 3 questionnaires including Back hopelessness scale, Kessler psychological Distress Scale, and Krupp the fatigue severity score. After 8 consecutive secession of ACT therapy in cases, both groups were compared in scores of aforementioned questionnaires before and after therapy. In case group, hopeless, psychological distress, and fatigue severity were improved significantly compared with controls $(\mathrm{p}<0.01)$. Few studies on this peruse have been conducted until recently. Acceptance and commitment therapy is a method in which patient learns to accept negative thoughts as a challenge not a believe. They learn to try to accept their limitations instead of fighting them. It is a method of psychological flexibility and motivation to find the best ways to overcome those limitations instead of denying them. This study demonstrated that ACT psychotherapy reduces frustration, psychological distress and mental tiredness in lupus patients.

\section{PS6:113 LONG-TERM HYDROXYCHLOROQUINE USE: ARE LUPUS PATIENTS UNDERMONITORED?: A UNIVERSITY TEACHING HOSPITAL EXPERIENCE}

K Sunmboye, S Shaffu, M McCartney. Rheumatology department University hospitals of Leicester, Leicester, UK

\subsection{6/lupus-2018-abstract.157}

Hydroxychloroquine is an integral part of maintenance therapy in lupus patients. Recent UK guidelines recommend that patients on hydroxychloroquine need to have regular monitoring. The risk of retinal toxicity becomes significant after a cumulative dose of 1 gram or after 7 years of drug use. Monitoring with use of spectral domain optical coherence tomography (OCT) is required after 5 years of use from recent guidelines.

The University hospitals of Leicester which serves 1 million population has a diverse group of caucasian and black with minority ethnic (BME) groups. There are 293 patients with lupus on the rheumatology databse of the tertiary hospital. $194(66 \%)$ of them are on hydroxychloroquine. We performed an audit of patients that have regular eye monitoring whilst on hydroxychloroquine. Letters were sent out to the patients with lupus on hydroxychloroquine. 106 responses were obtained with a response rate of 55\%. 96 (91\%) were female and the remainder were male. The average age of the respondents was 49 years. The average duartion of hydroxychloroquine use was 79 months. 53\% of the respondents had been on Hydroxychloroquine for more than 5 years. $42 \%$ of all respondents were made of patients in the BME group. Of the 106 responders, only $86 \%$ (91) were aware that eye checks were required. Majority of the patients (60\%) who were not aware of the need for regular eye monitoring were in the BME group. $71 \%$ of patients who had not had regular eye monitoring had other co-morbidities other than lupus. 1 in 5 of patients who did not have regular eye monitoring had other DMARDs alongside hydroxychloroquine. 20\% of this patient cohort were not compliant with regular eye monitoring after use of hydroxychloroquine after 5 years or more.

Ethnicity, disease factors, use of other DMARDs and duration of hydroxychloroquine use may play a role in determing patient compliance with regular eye monitoring. We recommended that more should be done to continually educate patients about the need to have yearly eye checks.

\section{PS6:114 HOW TO DIAGNOSE LUPUS ENTERITIS EARLY? LESSONS LEARNED FROM A MULTICENTER CASE SERIES}

${ }^{1} \mathrm{M}$ Luís, ${ }^{1} \mathrm{~L}$ Brites, ${ }^{2} \mathrm{AC}$ Duarte, ${ }^{3} \mathrm{~V}$ Teixeira, ${ }^{3} \mathrm{C}$ Macieira, ${ }^{2} \mathrm{MJ}$ Santos, ${ }^{1} \mathrm{~L}$ Inês. ${ }^{1} \mathrm{C}$ entro Hospitalar e Universitário de Coimbra - Department of Rheumatology, Coimbra, Portugal; ${ }^{2}$ Hospital Garcia de Orta - Department of Rheumatology, Lisboa, Portugal; ${ }^{3}$ Centro Hospitalar Lisboa Norte - Department of Rheumatology, Lisboa, Portugal

\subsection{6/lupus-2018-abstract.158}

Introduction Lupus enteritis (LE) is a rare, potentially lifethreatening manifestation of systemic lupus erythematosus (SLE). Early diagnosis is crucial for early treatment and prevention of serious complications such as ischaemic enteritis, bowel infarction with bleeding and/or perforation and peritonitis. The objective of this case review is to identify strategies for early diagnosis of LE.

Methods Retrospective analysis of patients with SLE (fulfilling ACR 1997 and/or SLICC classification criteria) and presenting LE from three tertiary SLE centres was conducted. The diagnosis was based on clinical and imaging features consistent with LE and exclusion of other causes of GI disorders.

Results We report six cases of LE (female: 100\%; age range: 16-55 years). All presented with acute onset abdominal pain, nausea and vomiting at the emergency department. One patient had lupus enteritis as inaugural manifestation of SLE. Of the remaining five, one presented at the previous visit to the lupus clinic with clinically active disease and two had serologically active/clinically inactive SLE. High anti-dsDNA antibodies and low serum complement were universally present at time of the LE event. Abdominal ultrasound was the first imaging exam to be performed in the emergency unit. In all cases it showed bowel wall thickening, dilatation of intestinal segments, increased reflectivity of mesenteric fat and mild ascites, raising the suspicion of LE and immediate start of treatment. These features were later confirmed by CT scan.

Discussion Abdominal ultrasound can be a reliable first line diagnostic tool for LE. It is a handy and radiation-free technique, an important advantage in women of child-bearing age.

\section{PS6:115 CYCLOPHOSPHAMIDE AS INDUCTION TREATMENT OF LUPUS NEPHRITIS IN A GROUP OF TUNISIAN PATIENTS: MONTHLY HIGH-DOSE OR BIWEEKLY LOW- DOSE PULSES?}

T Ben Salem, M Tougorti, M Lamloum, I Ben Ghorbel, MH Houman. Department of Internal Medicine, La Rabta University Hospital, Tunis, Tunisia

\subsection{6/lupus-2018-abstract.159}

Background Cyclophosphamide is an effective treatment of lupus nephritis (LN) but have many side effects especially with high doses. Two regimens are used: monthly high dose pulses or biweekly low dose pulses (Eurolupus protocol). LN 